\title{
ASYMPTOTIC TAIL PROBABILITIES FOR LARGE CLAIMS REINSURANCE OF A PORTFOLIO OF DEPENDENT RISKS
}

\section{BY}

\author{
Alexandru V. Asimit and Bruce L. Jones
}

\begin{abstract}
We consider a dependent portfolio of insurance contracts. Asymptotic tail probabilities of the ECOMOR and LCR reinsurance amounts are obtained under certain assumptions about the dependence structure.
\end{abstract}

\section{KEYWORDS}

Archimedean copula, Dependence, ECOMOR and LCR reinsurance, Tail probability.

\section{INTRODUCTION}

Insurance companies often use reinsurance as a mechanism for sharing risk, particularly when there is the possibility of catastrophic losses. Two appealing reinsurances are ECOMOR (excédent du coût moyen relatif) and LCR (largest claims reinsurance). Under ECOMOR, the reinsurer pays the sum of the exceedances of the $l$ largest claims over the $l+1$ st largest claim. Under LCR, the reinsurer pays the sum of the $l$ largest claims. ECOMOR and LCR treaties were proposed by Thépaut (1950) and Ammeter (1964), respectively.

We consider a portfolio of $n$ insurance contracts with associated loss random variables $X_{i}, i=1, \ldots, n$ assumed to be dependent. Let $X_{1, n} \geq \ldots \geq X_{n, n}$ be the corresponding upper order statistics. Then the reinsurance amounts under ECOMOR and LCR are given by

$$
E_{l}=\sum_{i=1}^{l}\left(X_{i, n}-X_{l+1, n}\right)
$$

and

$$
L_{l}=\sum_{i=1}^{l} X_{i, n}
$$

The purpose of this paper is to establish the asymptotic tail probabilities of the reinsurance amount under ECOMOR and LCR for a portfolio of dependent 
insurance contracts. This may be quite useful for risk management purposes, as it allows one to determine high quantiles of the reinsurance amount and therefore enables one to obtain capital amounts that will be adequate with high probability. This can also be done by performing a simulation study. However, to estimate high quantiles, a very large number of simulations are required, and since multivariate outcomes must be generated, the computations may be very time consuming.

\section{Preliminaries}

Let $Y_{i}, i=1,2, \ldots$ be a sequence of independent random variables with common distribution $F$, and let $M_{n}$ be the maximum of $Y_{1}, \ldots, Y_{n}$. If there exist constants $a_{n}, b_{n}$ and a random variable $Z$ with nondegenerate $\mathrm{df} G$ such that $a_{n} M_{n}+b_{n}$ converges weakly to $Z$, then $F$ is in the maximum domain of attraction of $G$ and we write $F \in \operatorname{MDA}(G)$. Moreover, by the Fisher-Tippett theorem (see, for example, Embrechts et al., 1997), $G$ belongs to the type of the distribution

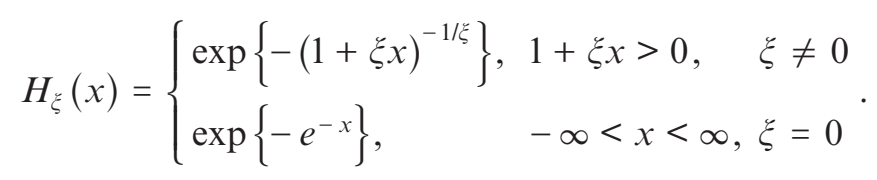

$H_{\xi}$ is known as the generalized extreme value distribution. For $\xi>0, \Phi_{\xi}(x):=$ $H_{1 / \xi}(\xi(x-1))$ is the standard Fréchet distribution, $\Psi_{\xi}(x):=H_{-1 / \xi}(\xi(x+1))$ is the standard Weibull distribution, and $\Lambda(x):=H_{0}(x)$ is the standard Gumbel distribution.

The dependence structure associated with the distribution of a random vector can be characterized in terms of a copula. An $n$-dimensional copula is a multivariate $\mathrm{df}$ defined on $[0,1]^{n}$ with uniformly distributed marginals. Due to Sklar's Theorem (see Sklar, 1959), if $X_{1}, \ldots, X_{n}$ has a joint distribution function with continuous marginals, then there exists a unique copula, $C$, such that

$$
\operatorname{Pr}\left(X_{1} \leq x_{1}, \ldots, X_{n} \leq x_{n}\right)=C\left(\operatorname{Pr}\left(X_{1} \leq x_{1}\right), \ldots, \operatorname{Pr}\left(X_{n} \leq x_{n}\right)\right) .
$$

Similarly, the survival copula, $\widehat{C}$, is defined as the copula relative to the joint survival function and satisfies

$$
\operatorname{Pr}\left(X_{1}>x_{1}, \ldots, X_{n}>x_{n}\right)=\widehat{C}\left(\operatorname{Pr}\left(X_{1}>x_{1}\right), \ldots, \operatorname{Pr}\left(X_{n}>x_{n}\right)\right) .
$$

A well-known class of copulas is the Archimedean class. By definition, an Archimedean copula $C$ is given by

$$
C\left(u_{1}, \ldots, u_{n}\right)=\varphi^{-1}\left(\sum_{i=1}^{n} \varphi\left(u_{i}\right)\right)
$$


where $\varphi:[0,1] \mapsto[0, \infty)$ is its generator. Some regularity conditions are necessary to ensure that $C$ is a valid copula (see Kimberling, 1974 and Nelsen, 1999, ch. 4).

An important concept that is crucial to establishing the main results of this paper is vague convergence. Let $\left\{\mu_{n}, n \geq 1\right\}$ be a sequence of measures on a locally compact Hausdorff space $\mathbb{E}$ with countable base. Then $\mu_{n}$ converges vaguely to some measure $\mu$ (written $\mu_{n} \stackrel{v}{\longrightarrow} \mu$ ) if for all continuous functions $f$ with compact support we have

$$
\lim _{n \rightarrow \infty} \int_{\mathbb{E}} f d \mu_{n}=\int_{\mathbb{E}} f d \mu .
$$

A thorough background on vague convergence is given by Kallenberg (1983) and Resnick (1987).

\section{Main Results}

Wüthrich (2003) and Alink et al. (2004 and 2005) consider the asymptotic tail behavior for a sum of dependent random variables when the survival copula is Archimedean. A similar problem is discussed by Albrecher et al. (2006), Barbe et al. (2006), Alink et al. (2007) and Kortschak and Albrecher (2007), when a more general dependence structure is assumed. Since the ECOMOR and LCR reinsurances are linear combinations of the order statistics, studying the asymptotic tail probability for the losses associated with these reinsurance treaties is closely related to the aforementioned problem.

The sufficient conditions of our main results imply that all individual losses are tail equivalent to a distribution function that is either in MDA of Fréchet or MDA of Gumbel. We now discuss these two cases.

\subsection{Results for MDA of Fréchet}

The next assumption is sufficient to establish our first main result.

Assumption 1. For any set $I \subseteq\{1, \ldots, n\}$,

$$
\lim _{t \rightarrow \infty} \frac{\operatorname{Pr}\left(X_{i}>t x_{i}, i \in I\right)}{\operatorname{Pr}\left(X_{1}>t\right)}
$$

exists and is finite for all $x_{i}>0$.

This assumption implies that the distribution functions are tail equivalent. This means that $0<\lim _{t \rightarrow \infty} \operatorname{Pr}\left(X_{i}>t\right) / \operatorname{Pr}\left(X_{1}>t\right)<\infty$ holds for any $i \in\{1, \ldots, n\}$. Moreover, there exists $\beta>0$ such that the distribution function of $X_{1}$ is in $\operatorname{MDA}\left(\Phi_{\beta}\right)($ see Resnick, 1987). 
Now, if the random variables $X_{1}, \ldots, X_{n}$ are exchangeable then,

$$
\begin{aligned}
& \operatorname{Pr}\left(X_{1, n}>t x_{1}, \ldots, X_{l, n}>t x_{l}\right) \\
& =\sum_{\left(k_{1}, \ldots, k_{l}\right) \in A_{l}} \frac{n !}{k_{1} ! \cdots k_{l} !\left(n-k_{1}-\cdots-k_{l}\right) !} \operatorname{Pr}\left(\left\{X_{1}, \ldots X_{k_{1}}>t x_{1}\right\},\right. \\
& \left.\quad\left\{t x_{2}<X_{k_{1}+1}, \ldots, X_{k_{1}+k_{2}} \leq t x_{1}\right\}, \ldots,\left\{X_{k_{1}+\ldots+k_{l}+1}, \ldots, X_{n} \leq t x_{l}\right\}\right),
\end{aligned}
$$

for any $x_{1}>\ldots>x_{l}$, where $A_{l}=\left\{\left(k_{1}, \ldots, k_{l}\right): i \leq k_{1}+\ldots+k_{i} \leq n, i=1, \ldots, l\right\}$. Each term on the right-hand side of (1) can be expressed as a linear combination of joint survival probabilities. This fact combined with Assumption 1 allows us to conclude that there exists a positive function $f_{l}$ such that

$$
\operatorname{Pr}\left(X_{1, n}>t x_{1}, \ldots, X_{1, n}>t x_{1}\right) \sim f_{l}\left(x_{1}, \ldots x_{l}\right) \operatorname{Pr}\left(X_{1}>t\right), t \rightarrow \infty .
$$

Under more general assumptions for which the exchangeability property does not hold, a similar but even more cumbersome relationship to that in (1) can be obtained.

Now, relation (2) implies that

$$
\frac{\operatorname{Pr}\left(\left(X_{1, n} / t, \ldots, X_{l, n} / t\right) \in \cdot\right)}{\operatorname{Pr}\left(X_{1}>t\right)} \stackrel{\bullet}{\rightarrow} \mu_{l}(\cdot)
$$

holds on $[0, \infty]^{l} \backslash\{\mathbf{0}\}$ where the measure $\mu_{l}$ is given by

$$
\mu_{1}\left(\left(x_{1}, \infty\right] \times \cdots \times\left(x_{1}, \infty\right]\right):=f_{l}\left(x_{1}, \ldots, x_{1}\right) .
$$

From the above and Proposition A2.12 of Embrechts et al. (1997 p. 563), we now have the essential development for the main results of this subsection, which are stated in the following theorem.

Theorem 1. Let $\left(X_{1}, \ldots, X_{n}\right)$ be a positive random vector such that Assumption 1 holds. For $l=1, \ldots, n-1$, the asymptotic tail probability for $E_{l}$, the reinsurance amount under an ECOMOR treaty, is given by

$$
\operatorname{Pr}\left(E_{l}>t\right) \sim C_{E F}(l, \alpha, \beta) \operatorname{Pr}\left(X_{1}>t\right) \text { as } t \longrightarrow \infty,
$$

where

$$
C_{E F}(l, \alpha, \beta)=\mu_{l+1}\left(\boldsymbol{x}: \sum_{i=1}^{l} x_{i}-l x_{l+1}>1, x_{1}>\cdots>x_{l+1}>0\right),
$$

with $\mu_{l}$ defined by (3). 
For $l=1, \ldots, n$, the asymptotic tail probability for $L_{l}$, the reinsurance amount under an LCR treaty, is given by

$$
\operatorname{Pr}\left(L_{l}>t\right) \sim C_{L F}(l, \alpha, \beta) \operatorname{Pr}\left(X_{1}>t\right) \text { as } t \longrightarrow \infty,
$$

where

$$
C_{L F}(l, \alpha, \beta)=\mu_{l}\left(\boldsymbol{x}: \sum_{i=1}^{l} x_{i}>1, x_{1}>\cdots>x_{l}>0\right) .
$$

It should be noted that in order to obtain these results, we used the fact that each measure $\mu_{l}$ has no mass on the boundaries of the sets used in defining the asymptotic constants, $C_{E F}$ and $C_{L F}$ (see Hult and Lindskog, 2002; and Kortschak and Albrecher, 2007).

\subsection{MDA of Gumbel}

As in the Fréchet case, the sufficient assumption from this subsection implies that the marginal distribution functions are tail equivalent and are from the MDA of Gumbel. It is well-known (see, for example, Embrechts et al., 1997) that if $F \in M D A(\Lambda)$, then there exists a positive, measurable function $a(\cdot)$ such that

$$
\lim _{t \rightarrow \infty} \frac{\bar{F}(t+x a(t))}{\bar{F}(t)}=e^{-x},
$$

for any real $x$. The sufficient condition of the next main result is now defined.

Assumption 2. For any set $I \subseteq\{1, \ldots, n\}$,

$$
\lim _{t \rightarrow \infty} \frac{\operatorname{Pr}\left(X_{i}>t+a(t) x_{i}, i \in I\right)}{\operatorname{Pr}\left(X_{1}>t\right)}
$$

exists and is finite for $-\infty<x_{i}<\infty$, where $a(\cdot)$ is the corresponding scaling function defined by (4) for the distribution function of $X_{1}$.

In the same manner as the previous subsection, we have

$$
\operatorname{Pr}\left(X_{1, n}>t+x_{1} a(t), \ldots, X_{l, n}>t+x_{1} a(t)\right) \sim \bar{F}(t) g_{l}\left(x_{1}, \ldots x_{1}\right),
$$

where $g_{l}$ is a positive function.

Now, relation (5) implies that

$$
\frac{\operatorname{Pr}\left(\left(X_{1, n}-t, \ldots, X_{l, n}-t\right) \in a(t) \cdot\right)}{\operatorname{Pr}\left(X_{1}>t\right)} \stackrel{\sim}{\rightarrow} v_{l}(\cdot)
$$


holds on $(-\infty, \infty]^{l}$ where the measure $v_{l}$ is given by

$$
v_{l}\left(\left(x_{1}, \infty\right] \times \cdots \times\left(x_{l}, \infty\right]\right):=g_{l}\left(x_{1}, \ldots, x_{1}\right) .
$$

Now, we are able to give the main result from this subsection, which is only for the LCR reinsurance. This is stated as Theorem 2, which is a consequence of Proposition A2.12 of Embrechts et al. (1997 p. 563).

Theorem 2. Let $\left(X_{1}, \ldots, X_{n}\right)$ be a positive random vector such that Assumption 2 holds. In addition, if there exists a positive function $V(\cdot)$ such that $g_{l}(\boldsymbol{x}+c \boldsymbol{1}) \geq$ $g_{l}(x) V(c)$ holds for any $\boldsymbol{x} \in \mathfrak{R}^{l}$ and $c>0$, where $l=1, \ldots, n$, then

$$
\operatorname{Pr}\left(L_{l}>l t\right) \sim C_{L G}(l, \alpha) \operatorname{Pr}\left(X_{1}>t\right) \text { as } t \longrightarrow \infty,
$$

where

$$
C_{L G}(l, \alpha)=v_{l}\left(\boldsymbol{x}: \sum_{i=1}^{l} x_{i}>0, x_{1}>\cdots>x_{l}\right),
$$

with $v_{l}$ defined by (6).

Two more remarks are useful in understanding Theorem 2. First, note that each measure $v_{l}$ has no mass on regions around $-\infty$. This is obvious for $l=1$, so we consider the case in which $l>1$. It is sufficient to check that

$$
\lim _{M \rightarrow \infty} v_{l}\left(\mathrm{x}: \sum_{i=1}^{l} x_{i}>0, x_{1}>\cdots>x_{l-1}>-M>x_{l}\right)=0 .
$$

In doing so, we first mention that the following clearly holds

$$
\begin{aligned}
\operatorname{Pr}\left(X_{1, n}>t\right) & =\left(\begin{array}{l}
n \\
1
\end{array}\right) \operatorname{Pr}\left(X_{1}>t\right)-\cdots+(-1)^{n+1}\left(\begin{array}{l}
n \\
n
\end{array}\right) \operatorname{Pr}\left(X_{1}>t, \ldots, X_{n}>t\right) \\
& \sim \Delta \operatorname{Pr}\left(X_{1}>t\right), \text { as } t \longrightarrow \infty,
\end{aligned}
$$

where the last step is due to Assumption 2 and $\Delta$ is a positive constant. Combining (4) and (8), we have

$$
\begin{gathered}
v_{l}\left(\mathrm{x}: \sum_{i=1}^{l} x_{i}>0, x_{1}>\cdots>x_{l-1}>-M>x_{l}\right) \\
\leq \lim _{t \rightarrow \infty} \frac{\operatorname{Pr}\left(X_{1, n}>t+a(t) \frac{M}{l-1}\right)}{\bar{F}(t)}=\Delta e^{-M /(l-1)},
\end{gathered}
$$


which leads to (7). Second, using the same reasoning as in the Fréchet case, the remainder of the boundary of the set used in defining the asymptotic constant $C_{L G}$ is $v_{l}$-negligible due to the fact that $g_{l}(\mathrm{x}+c \mathbf{1}) \geq g_{l}(\mathrm{x}) V(c)$.

\section{EXAMPLES}

Some multivariate distributions which satisfy the conditions imposed in Theorems 1 and 2 are now given. For ease of presentation, the assumption of identical marginals is made in all examples.

\subsection{Survival Archimedean}

The survival copula $\hat{C}$ is an Archimedean copula such that the generator $\varphi$ is regularly varying at 0 with index $-\alpha\left(\varphi \in R V_{-\alpha}^{0}\right)$. That is,

$$
\lim _{t \uparrow 0} \frac{\varphi(t x)}{\varphi(t)}=x^{-\alpha}
$$

for any positive $x$. For more details on regular variation, we refer the reader to Bingham et al. (1987).

The Clayton copula is an example of an Archimedean copula with generator, $\varphi(u)=u^{-\alpha}-1$, which satisfies the property $\varphi \in R V_{-\alpha}^{0}$. This copula has the form

$$
C\left(u_{1}, \ldots, u_{n}\right)=\left(1-n+\sum_{i=1}^{n} u_{i}^{-\alpha}\right)^{-1 / \alpha},
$$

where $\alpha>0$.

If $F \in \operatorname{MDA}\left(\Phi_{\beta}\right)$ and $\varphi \in R V_{-\alpha}^{0}$, then Assumption 1 is satisfied with

$$
\lim _{t \rightarrow \infty} \frac{\operatorname{Pr}\left(X_{1}>t x_{1}, \ldots, X_{l}>t x_{l}\right)}{\operatorname{Pr}\left(X_{1}>t\right)}=\left(\sum_{i=1}^{l} x_{i}^{\alpha \beta}\right)^{-1 / \alpha},
$$

For more details, see Alink et al. (2004).

If $F \in M D A(\Lambda)$ and $\varphi \in R V_{-\alpha}^{0}$, then Assumption 2 is satisfied with

$$
\lim _{t \rightarrow \infty} \frac{\operatorname{Pr}\left(X_{1}>t+x_{1} a(t), \ldots, X_{l}>t+x_{l} a(t)\right)}{\operatorname{Pr}\left(X_{1}>t\right)}=\left(\sum_{i=1}^{l} e^{\alpha x_{i}}\right)^{-1 / \alpha}
$$

For more details, see Alink et al. (2004). It is obvious that the other sufficient condition is satisfied with $V(c)=e^{-c}$. 


\subsection{Archimedean Copula}

The sufficient conditions from Theorems 1 and 2 are also satisfied if the actual copula is Archimedean such that the generator $\varphi$ is regularly varying at 1 . By definition, this means that for any positive $x$ the following holds

$$
\lim _{t \downarrow 0} \frac{\varphi(1-t x)}{\varphi(1-t)}=x^{\alpha}
$$

and we write $\varphi \in R V_{\alpha}^{1}$. Furthermore, the index satisfies the condition that $\alpha \geq 1$ (see Juri and Wütrich, 2003). The Gumbel copula is an example of such a copula with regularly varying generator $\varphi(u)=(-\ln u)^{\alpha}$, which satisfies the latter property $\left(\varphi \in R V_{\alpha}^{1}\right)$.

$$
C\left(u_{1}, \ldots, u_{n}\right)=\exp \left(-\left[\sum_{i=1}^{n}\left(-\ln u_{i}\right)^{\alpha}\right]^{1 / \alpha}\right),
$$

where $\alpha \geq 1$.

For this example, Assumption 1 holds with

$$
\lim _{t \rightarrow \infty} \frac{\operatorname{Pr}\left(X_{1}>t x_{1}, X_{2}>t x_{2}\right)}{\bar{F}(t)}=x_{1}^{-\beta}+x_{2}^{-\beta}-\left(x_{1}^{-\alpha \beta}+x_{2}^{-\alpha \beta}\right)^{1 / \alpha}, x_{1}, x_{2}>0,
$$

and Assumption 2 holds with

$$
\begin{aligned}
& \lim _{t \rightarrow \infty} \frac{\operatorname{Pr}\left(X_{1}>t+x_{1} a(t), X_{2}>t+x_{2} a(t)\right)}{\bar{F}(t)} \\
& \quad=e^{-x_{1}}+e^{-x_{2}}-\left(e^{-\alpha x_{1}}+e^{-\alpha x_{2}}\right)^{1 / \alpha},-\infty<x_{1}, x_{2}<\infty,
\end{aligned}
$$

provided that $1<\alpha<\infty$ (see Juri and Wütrich, 2003). For simplicity, the bivariate case has been considered, but the result can be extended to the multivariate case, which is more cumbersome. The remaining condition of the Gumbel case is obviously satisfied with $V(c)=e^{-c}$.

\section{3. t-copula}

A well known non-Archimedean copula is the t-copula. For the ease of presentation, we check the sufficient conditions only in the bivariate case. The bivariate $\mathrm{t}$-copula is given by

$$
C(u, v)=\int_{-\infty}^{t^{-1}(u ; a)} \int_{-\infty}^{t^{-1}(v ; a)} \frac{1}{2 \pi \sqrt{1-\rho^{2}}}\left(1+\frac{s^{2}-2 \rho s t+t^{2}}{\alpha\left(1-\rho^{2}\right)}\right)^{-1-\alpha / 2} d s d t
$$


where $t^{-1}(\cdot ; \alpha)$ is the inverse function of a standard univariate Student t-distribution with $\alpha$ degrees of freedom.

Simple computations yield

$$
\hat{C}(u x, u y) \sim u h(x / y) y, \text { as } u \downarrow 0,
$$

where

$$
h(x)=x \bar{T}_{\alpha+1}\left(\left(x^{1 / \alpha}-\rho\right) \frac{\sqrt{\alpha+1}}{\sqrt{1-\rho^{2}}}\right)+\bar{T}_{\alpha+1}\left(\left(x^{-1 / \alpha}-\rho\right) \frac{\sqrt{\alpha+1}}{\sqrt{1-\rho^{2}}}\right) .
$$

For more details, see Asimit and Jones (2007a). Note that we use the fact that $h(x)=x h(1 / x)$ holds. By using the same reasoning as Alink et al. (2007), one can establish

$$
\lim _{t \rightarrow \infty} \frac{\operatorname{Pr}\left(X_{1}>t x, X>t y\right)}{\operatorname{Pr}\left(X_{1}>t\right)}=h\left(x^{-\beta} y^{\beta}\right) y^{-\beta}
$$

when the distribution function of $X_{1}$ is in $\operatorname{MDA}\left(\Phi_{\beta}\right)$. Similarly, the expression in the Gumbel case is given by

$$
\lim _{t \rightarrow \infty} \frac{\operatorname{Pr}\left(X_{1}>t+a(t) x, X>t+a(t) y\right)}{\operatorname{Pr}\left(X_{1}>t\right)}=h\left(e^{-x+y}\right) e^{-y} .
$$

Once again, the other sufficient condition assumed in Theorem 2 is satisfied with $V(c)=e^{-c}$.

\subsection{A non-copula example}

The previous examples were given for some well-known copulas. It would be restrictive to limit our attention only to the cases for which the underlying copula is known. For this reason, an example is provided for which the copula does not have a closed form. Provided that the individual losses are identically distributed, the multivariate phase-type distribution satisfies the sufficient conditions of Theorem 2, at least for the bivariate case (see Asimit and Jones, 2007b). In this case, $g_{l}(\mathrm{x}+c \mathbf{1})=g_{l}(\mathrm{x}) V(c)$, and therefore the condition in Theorem 2 is satisfied with a constant $V(c)=e^{-c}$.

\section{NUMERICAL EXAMPLES}

In this section, examples for the asymptotic constants from Theorems 1 and 2 are given. In order to avoid long computations, a portfolio consisting of $n=3$ 
insurance contracts is considered. Explicit forms of the asymptotic constants are provided for $n=3$ and $l=2$, but numerical computations are made for higher dimensions. The required integrations were done using the numerical integration function in Mathematica. We consider the dependence structure discussed in Section 4.1. First, the Fréchet case is explored. From (1), we have

$$
\begin{aligned}
\operatorname{Pr}\left(X_{1,3}>t x_{1}, X_{2,3}>t x_{2}\right)=\operatorname{Pr}( & \left.X_{1}, X_{2}, X_{3}>t x_{1}\right)+3 \operatorname{Pr}\left(X_{1}, X_{2}>t x_{1}, X_{3} \leq t x_{2}\right) \\
+ & 3 \operatorname{Pr}\left(X_{1}, X_{2}>t x_{1}, t x_{2}<X_{3} \leq t x_{1}\right) \\
+ & 3 \operatorname{Pr}\left(X_{1}>t x_{1}, t x_{2}<X_{2}, X_{3} \leq t x_{1}\right) \\
& +6 \operatorname{Pr}\left(X_{1}>t x_{1}, t x_{2}<X_{2} \leq t x_{1}, X_{3} \leq t x_{2}\right),
\end{aligned}
$$

for any $x_{1}>x_{2}>0$. Otherwise,

$$
\operatorname{Pr}\left(X_{1,3}>t x_{1}, X_{2,3}>t x_{2}\right)=\operatorname{Pr}\left(X_{1}, X_{2}, X_{3}>t x_{2}\right)+3 \operatorname{Pr}\left(X_{1}, X_{2}>t x_{2}, X_{3} \leq t x_{2}\right) .
$$

Straightforward computations together with (9) yield the following

$$
f_{2}\left(x_{1}, x_{2}\right)=\left\{\begin{array}{cc}
\left(3^{-1 / \alpha}-3 \cdot 2^{-1 / \alpha}\right) x_{1}^{-\beta}+6\left(x_{1}^{\alpha \beta}+x_{2}^{\alpha \beta}\right)^{-1 / \alpha} & \\
-3\left(x_{1}^{\alpha \beta}+2 x_{2}^{\alpha \beta}\right)^{-1 / \alpha}, & 0<x_{2}<x_{1} . \\
\left(3 \cdot 2^{-1 / \alpha}-2 \cdot 3^{-1 / \alpha}\right) x_{2}^{-\beta}, & 0<x_{1} \leq x_{2}
\end{array}\right.
$$

In a similar manner, if $F \in M D A(\Lambda)$ then (10) yields

$$
g_{2}\left(x_{1}, x_{2}\right)=\left\{\begin{array}{cc}
\left(3^{-1 / \alpha}-3 \cdot 2^{-1 / \alpha}\right) e^{-x_{1}}+6\left(e^{\alpha x_{1}}+e^{\alpha x_{2}}\right)^{-1 / \alpha} & \\
-3\left(e^{\alpha x_{1}}+2 e^{\alpha x_{2}}\right)^{-1 / \alpha}, & 0<x_{2}<x_{1} . \\
\left(3 \cdot 2^{-1 / \alpha}-2 \cdot 3^{-1 / \alpha}\right) e^{-x_{2}}, & 0<x_{1} \leq x_{2}
\end{array}\right.
$$

It follows from Theorem 1 that

$$
\begin{aligned}
& C_{E F}(1, \alpha, \beta) \\
& \quad=\mu_{2}\left(\left(x_{1}, x_{2}\right): x_{1}-x_{2}>1,0<x_{2}<x_{1}\right) \\
& \quad=6 \beta \int_{0}^{\infty} t^{\alpha \beta-1}\left\{\left[t^{\alpha \beta}+(1+t)^{\alpha \beta}\right]^{-1-1 / \alpha}-\left[2 t^{\alpha \beta}+(1+t)^{\alpha \beta}\right]^{-1-1 / \alpha}\right\} d t
\end{aligned}
$$

and 


$$
\begin{aligned}
C_{L F}(2, \alpha, \beta) \\
=\mu_{2}\left(\left(x_{1}, x_{2}\right): x_{1}+x_{2}>1,0<x_{2}<\mathrm{x}_{1}\right) \\
=6(1+\alpha) \beta^{2} \int_{1 / 2}^{1} \int_{1-s}^{s}(s t)^{\alpha \beta-1}\left[\left(s^{\alpha \beta}+t^{\alpha \beta}\right)^{-2-1 / \alpha}-\left(s^{\alpha \beta}+2 t^{\alpha \beta}\right)^{-2-1 / \alpha}\right] d t d s \\
\quad+6(1+\alpha) \beta^{2} \int_{1}^{\infty} \int_{0}^{s}(s t)^{\alpha \beta-1}\left[\left(s^{\alpha \beta}+t^{\alpha \beta}\right)^{-2-1 / \alpha}-\left(s^{\alpha \beta}+2 t^{\alpha \beta}\right)^{-2-1 / \alpha}\right] d t d s \\
=3-3 \cdot 2^{-1 / \alpha}+3^{-1 / \alpha} \\
\quad+6(1+\alpha) \beta^{2} \int_{1 / 2}^{1} \int_{1-s}^{s}(s t)^{\alpha \beta-1}\left[\left(s^{\alpha \beta}+t^{\alpha \beta}\right)^{-2-1 / \alpha}-\left(s^{\alpha \beta}+2 t^{\alpha \beta}\right)^{-2-1 / \alpha}\right] d t d s,
\end{aligned}
$$

where $\mu_{2}\left(\left(x_{1}, \infty\right] \times\left(x_{2}, \infty\right]\right):=f_{2}\left(x_{1}, x_{2}\right)$. Also, from Theorem 2,

$$
\begin{aligned}
& C_{L G}(2, \alpha) \\
& \quad=v_{2}\left(\left(x_{1}, x_{2}\right): x_{1}+x_{2}>0, x_{1}>x_{2}\right) \\
& \quad=6(1+\alpha) \int_{0}^{\infty} \int_{-s}^{s} e^{\alpha(s+t)}\left[\left(e^{\alpha s}+e^{\alpha t}\right)^{-2-1 / \alpha}-\left(e^{\alpha s}+2 e^{\alpha t}\right)^{-2-1 / \alpha}\right] d t d s,
\end{aligned}
$$

where $v_{2}\left(\left(x_{1}, \infty\right] \times\left(x_{2}, \infty\right]\right):=g_{2}\left(x_{1}, x_{2}\right)$.

Numerical exemplifications of our main results are now considered for the LCR treaty. It is assumed that each marginal is a two-parameter Pareto distribution with df

$$
F_{\text {Pareto }}(x ; \beta, \gamma)=1-\left(1+\gamma \frac{x}{\beta}\right)^{-\beta}, x \geq 0
$$

in order to illustrate Theorem 1 and exponentially distributed for Theorem 2 . In both cases, the expected value is set to 10,000 , which implies that the Pareto parameters should satisfy $\gamma=\beta /((\beta-1) \times 10,000)$. We performed the calculations for a variety of $\alpha$ and $\beta$ values. Tables 1 to 3 show the values of the asymptotic constants and the resulting quantiles at level 0.999. Table 2 shows that, in the Fréchet case, changing the value of $\alpha$ does not have a significant impact on the quantile, but the sensitivity to $\beta$ is quite apparent. Similarly, table 3 shows that, in the Gumbel case, the value of $\alpha$ does not significantly influence the quantile.

\section{ACKNOWLEDGMENTS}

The authors wish to thank an anonymous referee for helpful comments which improved the paper. This work was supported by a Discovery Grant from the Natural Sciences and Engineering Research Council of Canada. 
TABLE 1

Asymptotic Constants $C_{L F}(l, \alpha, \beta)$.

$$
l=2
$$

\begin{tabular}{r|rcc|ccc|ccc}
\hline & \multicolumn{3}{|c|}{$\beta=1$} & \multicolumn{3}{c|}{$\beta=3$} & \multicolumn{3}{c}{$\beta=5$} \\
\hline$\alpha$ & $n=2$ & $n=3$ & $n=4$ & $n=2$ & $n=3$ & $n=4$ & $n=2$ & $n=3$ & $n=4$ \\
\hline 1 & 2.00 & 2.67 & 3.17 & 6.84 & 9.82 & 12.0 & 26.4 & 38.6 & 47.3 \\
3 & 2.00 & 2.31 & 2.49 & 7.81 & 9.13 & 9.88 & 31.1 & 36.4 & 39.5 \\
5 & 2.00 & 2.20 & 2.30 & 7.92 & 8.75 & 9.20 & 31.6 & 35.0 & 36.8 \\
10 & 2.00 & 2.10 & 2.16 & 7.98 & 8.41 & 8.62 & 31.9 & 33.6 & 34.5 \\
\hline \hline
\end{tabular}

\begin{tabular}{r|cc|cc|cc}
\hline \hline \multicolumn{1}{c}{$l=3$} \\
\hline & \multicolumn{2}{|c|}{$\beta=1$} & \multicolumn{2}{c}{$\beta=3$} & \multicolumn{2}{c}{$\beta=5$} \\
\hline$\alpha$ & $n=3$ & $n=4$ & $n=3$ & $n=4$ & $n=3$ & $n=4$ \\
\hline 1 & 3.00 & 3.75 & 21.8 & 29.8 & 186 & 261 \\
3 & 3.00 & 3.37 & 26.1 & 29.8 & 234 & 268 \\
5 & 3.00 & 3.24 & 26.7 & 29.0 & 239 & 261 \\
10 & 3.00 & 3.13 & 26.9 & 28.1 & 242 & 253 \\
\hline \hline
\end{tabular}

TABLE 2

Quantile Estimates of $L_{2}$ AT 0.999 LeVel Based on Theorem 1.

\begin{tabular}{r|c|c|c|c}
\hline \hline$\alpha$ & $\beta=2$ & $\beta=3$ & $\beta=4$ & $\beta=5$ \\
\hline 1 & 698,462 & 408,318 & 324,199 & 290,675 \\
3 & 666,514 & 397,994 & 318,609 & 286,866 \\
5 & 651,930 & 392,197 & 315,049 & 284,226 \\
10 & 638,451 & 386,682 & 311,612 & 281,654 \\
\hline \hline
\end{tabular}

TABLE 3

Asymptotic Constants $C_{L G}(2, \alpha)$ and Quantile Estimates of $L_{2}$ AT LeVel 0.999.

\begin{tabular}{r|c|c}
\hline \hline$\alpha$ & $C_{L G}(2, \alpha)$ & Quantile \\
\hline 1 & 1.18 & 141,402 \\
3 & 1.14 & 140,688 \\
5 & 1.09 & 139,918 \\
10 & 1.05 & 139,139 \\
\hline \hline
\end{tabular}




\section{REFERENCES}

Albrecher, H., Asmussen, S. and Kortschak, D. (2006) "Tail Asymptotics for the Sum of Two Heavy-Tailed Dependent Risks", Extremes, 9(2), 107-130.

Alink, S., Löwe, M. and WÜthrich, M.V. (2004) "Diversification of Aggregate Dependent Risks", Insurance: Mathematics and Economics, 35(1), 77-95.

Alink, S., Löwe, M. and Wüthrich, M.V. (2005) "Analysis of the Expected Shortfall of Aggregate Dependent Risks", ASTIN Bulletin, 35(1), 25-43.

Alink, S., Löwe, M. and Wüthrich, M.V. (2007) "Diversification for General Copula Dependence", Statistica Neerlandica, 61(4), 446-465.

Ammeter, H. (1964) "The Rating of Largest Claim Reinsurance Covers", Quarterly Letter from the Algemeine Reinsurance Companies Jubilee, 2, 5-17.

Asimit, A.V. and Jones, B.L. (2007a) "Extreme Behavior of Bivariate Elliptical Distributions", Insurance: Mathematics and Economics, 41(1), 53-61.

Asimit, A.V. and Jones, B.L. (2007b) "Extreme Behavior of Multivariate Phase-type Distributions", Insurance: Mathematics and Economics, 41(2), 223-233.

Barbe, P., Fougères, A.-L. and Genest, C. (2006) "On the Tail Behavior of Sums of Dependent Risks", ASTIN Bulletin, 36(2), 361-373.

Bingham, N.H., Goldie, C.M., and Teugels, J.L. (1987) Regular Variation. Cambridge University Press, Cambridge.

Embrechts, P., KlüPPelBerg, C. and Mikosch, T. (1997) Modelling Extremal Events for Insurance and Finance. Springer-Verlag, Berlin.

Hult, H. and Lindskog, F. (2002) "Multivariate Extremes, Aggregation and Dependence in Elliptical Distributions", Advances in Applied Probability, 34(3), 587-608.

JuRI, A. and WÜtRICH, M.V. (2003) "Tail dependence from a distributional point of view", Extremes, 6(3), 213-246.

KAllenberg, O. (1983) Random Measures, 3rd edition Akademie-Verlag, Berlin.

Kimberling, C.H. (1974) "A Probabilistic Interpretation of Complete Monotonicity", Aequationes Mathematica, 10, 152-164.

KortschaK, D. and Albrecher, H. (2007) "Asymptotic Results for the Sum of Dependent Non-identically Distributed Random Variables", Methodology and Computing in Applied Probability, to appear.

Nelsen, R.B. (1999) An Introduction to Copulas. Springer-Verlag, New York.

Resnick, S.I. (1987) Extreme Values, Regular Variation and Point Processes. Springer-Verlag, New York.

Sklar, A. (1959) "Fonctions de répartion à n dimensions et leurs marges", Publications de l'Institut de Statistique de l'Université de Paris, 8, 229-231.

ThÉPAuT, A. (1950) "Une nouvelle forme de réassurance: le traité d'excédent de coût moyen relatif (ECOMOR)", Bulletin Trimestriel de l'Institut des Actuaries Français, 49, 273-343.

Wüthrich, M.V. (2003) "Asymptotic Value-at-Risk Estimates for Sums of Dependent Random Variables", ASTIN Bulletin, 33(1), 75-92.

Alexandru V. Asimit

Department of Statistics - University of Toronto

100 St. George St. - Toronto, Ontario, Canada M5S $3 G 3$

E-mail:vali@utstat.toronto.edu

BRUCE L. JONES

Department of Statistical and Actuarial Sciences - University of Western Ontario London, Ontario - Canada N6A $5 B 7$

Tel.: 519-661-3149

Fax: 519-661-3813

E-mail: jones@stats.uwo.ca 